MS04-P07

\section{Structural basis for transcriptional control of flagellum assembly by the FliD-FliT complex}

\author{
Hyung Ho Lee ${ }^{1}$ \\ 1. Department of Chemistry, Seoul National University, Seoul, Korea \\ South \\ email: lee.hyungho@gmail.com
}

During the stages of the flagellar assembly process, the flagellar genes are expressed in response to morphological development of flagellar assembly. Flagellar biogenesis is controlled by a negative feedback loop. When FliD was secreted at the late step of flagellar assembly, the FliD-FliT complex disassembled and free FliT bound to the FlhDC complex, a master regulator of flagellar biogenesis, subsequently inhibiting the overall expression of flagellar proteins. Thus, the overall production of flagellar proteins is reduced by sensing flagellar morphology via FliD-FliT complex. In this study, we analyzed the role of the FliD C-terminal domain in pentamer formation and interaction with FliT. We determined the crystal structure of C-terminal domain of FliD bound to FliT, and showed that the FliD Leu443, which is part of the hydrophobic core of the FliDFliT complex, plays a crucial role in the pentameric oligomerization of FliD. When the FliD Leu443 was mutated, the binding affinity between FliD and FliT was significantly reduced, and it exists as a monomer in solution. Consistently, lengths of flagella in each cell were significantly reduced in L443R mutant strain, suggesting that normal flagellar biogenesis was impeded. These results suggest that the C-terminal domain of FliD plays a crucial role in the pentameric oligmerization of FliD and the binding of FliT to the C-terminal domain of FliD is critical to inhibit the premature assembly of the FliD pentamer in the cytosol.

References:

[1] Kim H. MJ.; Yoo, W.; Jin, K. S.; Ryu, S.; Lee, H. H. The role of the FliD C-terminal domain in pentamer formation and interaction with FliT. Scientific Reports. 2017. 7, 4418

Keywords: Flagellum, FliD, FliT

\section{MS04-P08}

\section{Liquid Dense Clusters: Intermediates between Nanocrystals and Single Particles}

Hevila Brognaro ${ }^{1}$, Sven Falke ${ }^{2}$, Robin Schubert ${ }^{2}$, Christian Betzel ${ }^{2}$

1. Center for Free Electron Laser Science. Deutsches ElektronenSynchrotron (DESY)., Hamburg, Germany

2. Laboratory for Structural Biology of Infection and Inflammation, c/o DESY, Institute for Biochemistry and Molecular Biology,

University of Hamburg, Hamburg, Germany

email: hevila.brognaro@desy.de

Liquid-dense clusters (LDCs) are formed by liquid-liquid phase separation, which is a process known for a long time in vitro as well as in vivo affecting a variety of nucleic acids, protein functions and metabolic pathways. Insights in the formation and dynamics of liquid dense clusters will open also new option to optimize protein crystal growth [1].

Nonetheless, the knowledge about structural and dynamic properties of macromolecule LDCs of proteins and different nucleic acids remains by far incomplete (1). Insights about the folding state and packing of biomolecules inside LDCs will aid in understanding the relevance of metastable liquid-liquid phase separation, as well as understanding initial processes of protein aggregation, oligomerization and misfolding, e.g. in neurodegenerative diseases like Alzheimer, Parkinson, dementia and sclerosis [2]. In order to investigate formation and internal structure of liquid dense clusters (LDC) by coherent X-ray diffractive imaging, selected distinct proteins of high medical and biological relevance as well as RNAs were selected to prepare LDCs. The physico-chemical conditions for the formation, stabilization and labelling of selected macromolecule LDCs were established and systematically optimized. The influence of polyvalent ions and polyethylene glycols as precipitant agents, which can promote LDC formation, were investigated. A combination of single particle Brownian microscopy applying an ultrafast camera, in situ Dynamic Light Scattering as well as fluorescens and atomic force microscopy have been applied to follow the time resolved process of clusters formation. Initial results and data obtained were utilized to understand and to optimize the clusters formation, as well as assess their stability, also towards advanced X-ray imaging techniques. More detailed information about folding state and arrangement of macromolecules inside LDCs, particularly by using X-ray free electron laser based imaging will open new routes to utilize LDCs for structural investigation of nanomaterials and single macromolecules in a non-crystalline environment in the future, and will allow to prepare distinct nano- and micro-sized crystals, most suitable for serial diffraction data collection. Details will be presented.

References:

[1] Falke, S. et al. (2018). Enc Anal Chem. Accepted.

[2] B. Maziuk. et al. (2017). Front Mol Neurosci.10, 89.

Keywords: liquid dense clusters, crystal nucleation 